contrast to random synthetic networks, the number of connections is not the only indicator of high spreading capacity. Instead, the whole network topology must be taken into account in the identification of super-spreaders.

By means of large-scale numerical simulations performed on a set of representative real networks, Kitsak et al. ${ }^{1}$ pointed out that the highest efficiency in the spread of a disease is actually associated with nodes belonging to the most densely connected core of the network, as defined in terms of a $k$-core decomposition ${ }^{4}$. The authors argued that this core is formed by a subset of highly interconnected nodes - allowing for the presence of many infection pathways among them - and also connecting them to the rest of the network. In this way, an outbreak starting inside the core has a very strong chance of infecting the whole core and, by extension, reaching a sizeable fraction of the whole population.

The key point at play here is that, in real networks, not all highly connected nodes belong to the core. Therefore, even if an individual with high connectivity facilitates a large number of secondary infections, its effect on the network will not extend further if it is not part of the core. In the case of random synthetic networks, this situation is negligible, because all highly connected nodes usually belong to the core.

The results reported by Kitsak et al. ${ }^{1}$ have important consequences from a practical point of view. Indeed, given the intrinsic weakness that heterogeneous social networks show in terms of epidemic spreading, one of the first issues considered theoretically in response to this finding was the design of optimized immunization strategies. Standard medical policy consists of vaccinating everyone at an early age. Although this practice is the most rational and just one, it can be impossible to apply in practice due to limited economic resources. The question is then how to select a small number of people inside a population in such a way as to greatly reduce the global impact of a disease in the population through the herd-immunity effect.

Initial studies pointed to immunization of hubs as an optimal strategy, which can be found even in the absence of global topological knowledge by a clever trick ${ }^{5}$. Other, more effective and sophisticated approaches have been subsequently proposed, based on vaccination of the most central individuals ${ }^{6}$. The findings of Kitsak et al. ${ }^{1}$ hinted that aiming vaccination campaigns at individuals in the network core might be more effective.

From a more theoretical point of view, the observations of Kitsak et al. ${ }^{1}$, when coupled with exact results concerning the threshold in heterogeneous networks, have led to the discovery of the existence of different activation mechanisms for epidemics in heterogeneous networks. In these mechanisms, it is either the whole core, or the hubs by themselves, that are capable of sustaining a long-lived epidemic steady state ${ }^{7}$.

Turning back to our initial consideration, it is interesting to recall that spreading phenomena can be regarded from a more general perspective, by shifting focus from the transmission of biological diseases to the social propagation of rumours, information or memes ${ }^{8}$. In this case we might not be looking to hinder the spreading, but rather to enhance it, to reach the largest fraction of the population in the shortest possible time.

According to the results of Kitsak et al. ${ }^{1}$, an optimal marketing strategy should aim to first infect those individuals deep in the core of the social network with a new meme - and not those that simply have more friends or contacts. This insight, however, seems to be in opposition to recently published studies on the dynamics of rumour spreading in social networks ${ }^{9}$, which identify individuals in the core as better hinderers than enhancers of rumour propagation. It is clear we've still some way to go before we fully understand this idea and the manifold possible applications it implies.

Romualdo Pastor-Satorras is in the Departament de Física i Enginyeria Nuclear, Universitat Politécnica de Catalunya, 08034 Barcelona, Spain.

e-mail:romualdo.pastor@upc.edu

References

1. Kitsak, M. et al. Nature Phys. 6, 888-893 (2010).

2. Newman, M. E. J. Networks: An Introduction (Oxford Univ Press, 2010).

3. Pastor-Satorras, R. \& Vespignani, A. Phys. Rev. Lett. 86, 3200-3203 (2001).

4. Seidman, S. B. Social Networks 5, 269 -287 (1983).

5. Cohen, R., Havlin, S. \& ben-Avraham, D. Phys. Rev. Lett. 91, 247901 (2003).

6. Holme, P., Kim, B. J., Yoon, C. N. \& Han, S. K. Phys. Rev. E 65, 056109 (2002)

7. Castellano, C. \& Pastor-Satorras, R. Sci. Rep. 2, 371 (2012)

8. Daley, D. J. \& Kendall, D. G. Nature 204, 1118 (1964)

9. Borge-Holthoefer, J. \& Moreno, Y. Phys. Rev. E 85, $026116(2012)$

\title{
To the extreme
}

High-harmonic spectroscopy is a powerful tool for probing the electronic structure of atoms and molecules in gases. Experiments now show that similar emission from solids has a different origin.

\section{Giulio Vampa and David M. Villeneuve}

t has been 54 years since Peter Franken and co-workers ${ }^{1}$ first observed the generation of light in a crystal at the second harmonic of the incoming laser frequency - famously, the production staff at Physical Review Letters removed what they thought was an imperfection on the photograph of the spectrum, removing the weak second harmonic emission from the published article. Nevertheless, this experiment ushered in the era of nonlinear optics, made possible by the invention of what Franken called the optical maser, but what is known today as the laser.

Researchers at the Max Planck Institute for Quantum Optics, in Garching, Germany, have taken Franken's experiment to the next level. In a Letter published in Nature, Luu et al. ${ }^{2}$ report the observation of coherent extreme ultraviolet radiation emitted from the bulk of fused silica, which corresponds to the 17th harmonic of the incoming laser frequency.

To avoid melting the fused silica while maintaining the high intensity required to generate high photon energies of up to $30 \mathrm{eV}$, Luu et al. used an optical frequency synthesizer ${ }^{3}$ to sculpt the electrical waveform of the laser pulse. The resulting pulses had durations of 


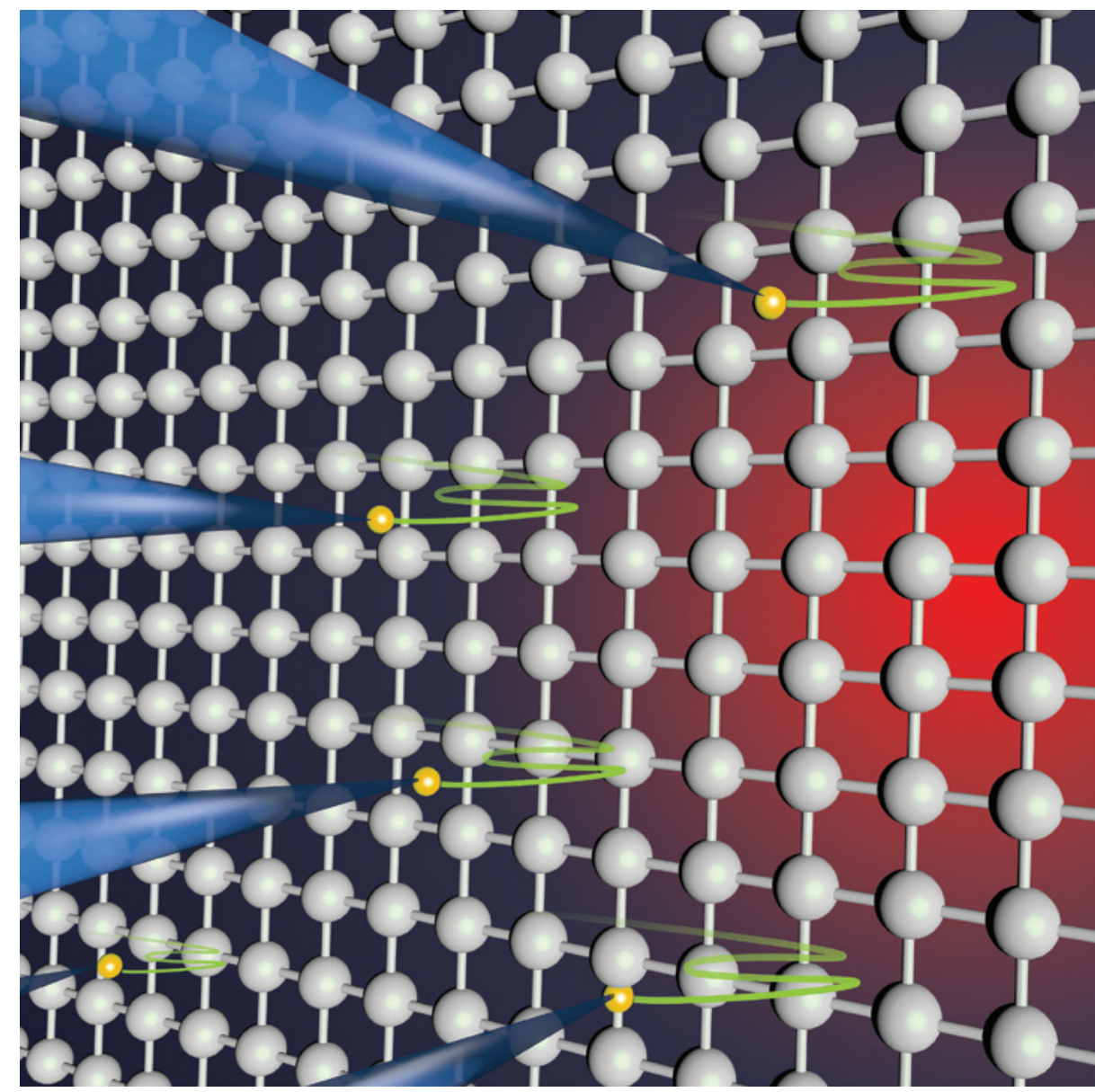

Figure 1 | High-harmonic generation in solids. An incoming laser beam (red) drives electrons (yellow) away from their parent atoms. The combination of this driving force with the restoring force from the lattice potential sets up electron trajectories (green) that result in the emission of high-harmonic radiation in the extreme ultraviolet (blue)

just a few femtoseconds and had optical bandwidths that covered the ultraviolet and near-infrared spectral regions. In the fused silica, the laser produced electric fields of over $1 \mathrm{~V}^{-1}$, rivalling the atomic fields that hold the electrons in place in the crystal lattice.

The main problem addressed by Luu et al. is the determination of the exact mechanism by which the electrons respond to the intense field. When a low-intensity optical field is applied to a dielectric material, the electrons move sinusoidally in synchrony with the field. This is described as a polarization of the medium, and defines the index of refraction of the material. As the laser intensity is increased, the electrons no longer respond sinusoidally and the instantaneous polarization contains new frequencies, just as a strongly driven guitar string emits overtones of its natural frequency.

This is not surprising on its own: conventional nonlinear optics can be understood in terms of an electron oscillating in the anharmonic potential of the ion to which it is bound. The force here, however, is so strong that it detaches the electron from the ion, leaving it oscillating across many lattice sites. Luu et al. calculate that the electron can travel as far as $30 \AA$ ! But the electron is not 'free', as it is still subjected to the potential of the lattice. When the de Broglie wavelength of the electron becomes comparable to the interatomic separation, a portion of the forward propagating wave is backreflected, and the electron is decelerated. The lattice therefore opposes the fielddriven oscillation, making the electron's motion anharmonic. Luu et al. show that surprisingly high photon energies are emitted in this process.

The emission of harmonics of a laser frequency from a solid is similar to the more familiar emission of harmonics from a gas driven by an intense femtosecond laser pulse. This is called high-harmonic generation, and is the basis of the field of attosecond science. In gas-phase highharmonic generation, the intense laser field removes an electron from each atom and drives it back to its parent ion (the 'hole' in the language of solids), where it recombines and releases the kinetic energy gained in the laser field through the emission of an energetic photon (Fig. 1).

According to the results of Luu et al., a similar mechanism doesn't seem to be happening in solids. The experiment can be understood by considering the motion of electrons tunnelled into the conduction band alone. This current-driven emission is a unique feature of solids, because electrons in vacuum will always move synchronously with the field. Luu et al. take a step forward in the comparison with the atomic counterpart by comparing the total photon yield of fused silica and two noble gases, Ar and $\mathrm{Kr}$. One would expect that the far higher density of the solid would result in much stronger extreme ultraviolet emission, but they find the emission from the solid to be only eight times greater than from the gases.

As the electron oscillates coherently with the field, it returns information about the structure of the band in which it moves. Luu et al. demonstrate that the emission is sensitive to high spatial frequencies in the band structure. It is the first time that an alloptical technique can offer a glimpse at such elusive properties of solids.

The reach of extreme nonlinear optics has clearly been broadened. This experiment shows that femtosecond laser techniques can be applied to condensed matter to shed light on the attosecond response of electrons in a crystal lattice. Perhaps manipulating the coherent electron motion in the solid will permit us to gain new information on the intrinsic properties of solids, such as their band structure, particularly when strongly driven. The different origin of high-harmonic emission, current-driven as opposed to re-collision based, limits the borrowing of spectroscopic techniques from gas-phase research. New tools will have to be developed.

Giulio Vampa and David M. Villeneuve are in the Joint Attosecond Science Laboratory, National Research Council and University of Ottawa,

100 Sussex Drive, Ottawa, Ontario K1A 0R6, Canada. e-mail:gvamp015@uottawa.ca; david.villeneuve@nrc-cnrc.gc.ca

\section{References}

1. Franken, P. A., Hill, A. E., Peters, C. W. \& Weinreich, G Phys. Rev. Lett. 7, 118 (1961).

2. Luu, T. T. et al. Nature 521, 498-502 (2015).

3. Hassan, M. Th. et al. Rev. Sci. Instrum. 83, 111301 (2012).

Published online: 22 June 2015 